\title{
Prospective randomized double-blind study of efficacy and safety of 1c class antiarrhythmic agent (propafenone) for supraventricular arrhythmias in septic shock compared to amiodarone
}

Martin Balik ( $\square$ martin.balik@vfn.cz )

1st Medical Faculty Charles University https://orcid.org/0000-0003-1864-2143

Petr Waldauf

1st Medical Faculty Charles University

Michal Maly

1st Medical Faculty Charles University

Vojtech Matousek

1st Medical Faculty Charles University

Tomas Brozek

1st Medical Faculty Charles University

Jan Rulisek

1st Medical Faculty Charles University

Michal Porizka

1st Medical Faculty Charles University

Robert Sachl

1st Medical Faculty Charles University

PetrBrestovansky

1st Medical Faculty Charles University

Michal Otahal

1st Medical Faculty Charles University

Eva Svobodova

1st Medical Faculty Charles University

Marek Flaksa

1st Medical Faculty Charles University

Zdenek Stach

1st Medical Faculty Charles University

Jaroslav Pazout

1st Medical Faculty Charles University

Frantisek Duska

1st Medical Faculty Charles University 
Ondrej Smid

1st Medical Faculty Charles University

Martin Stritesky

1st Medical Faculty Charles University

\section{Method Article}

Keywords: Supraventricular arrhythmia, septic shock, propafenone, amiodarone, intensive care Posted Date: February 8th, 2019

DOI: https://doi.org/10.21203/rs.2.312/v1

License: (c) (1) This work is licensed under a Creative Commons Attribution 4.0 International License. Read Full License 


\section{Abstract}

Background: Septic shock often leads to supraventricular arrhythmias which contribute to haemodynamic compromise. A large retrospective study in this population generated the hypothesis that propafenone could be more effective than amiodarone in achieving and maintaining sinus rhythm in new-onset supraventricular arrhythmia. Moreover, the success of cardioversion can be predicted by certain echocardiographic parameters, which can guide the decision whether to aim for rhythm or rate control.

Methods: A prospective double-blind multi-center randomized controlled trial includes patients with newonset arrhythmia related to septic shock (2016 definition), but without severe impairment of the left ventricular ejection fraction. After baseline echocardiography, the patient will be randomised to receive a bolus and maintenance dose of either amiodarone or propafenone. The primary outcome is the proportion of patients that have achieved rhythm control at 24 hours after the start of the infusion. The secondary outcomes are the composite percentage of patients that needed rescue treatments (DC cardioversion or unblinding and cross over of the antiarrhythmics) within 24 hours, recurrence of arrhythmias, ICU mortality, 28-day and 1-year mortality. In the post-hoc analysis we plan to separately assess subgroups of patients with pulmonary hypertension and right ventricular dysfunction without left ventricular systolic dysfunction. In the exploratory part of the study we will assess whether (1.) the presence of a transmitral diastolic A wave and its higher velocity-time integral is predictive for the sustainability of mechanical sinus rhythm and whether (2.) the indexed left atrial endsystolic volume is predictive of recurrent arrhythmia.

Discussion: Amiodarone has become the first-line agent of use in almost any tachyarrhythmia in the critically ill. Nevertheless, it has a wide range of side effects and may not be the most effective drug in all circumstances. In light of this, we designed a prospective randomised controlled trial. Considering that in the observational study the restoration of sinus rhythm within $24 \mathrm{~h}$ occurred in $74 \%$ of the amiodaronetreated patients and in $89 \%$ of patients treated with propafenone, we plan to include 200 patients to have an $80 \%$ chance to demonstrate the superiority of propafenone at $p=0.05$. Assuming a $10 \%$ dropout, we plan to randomize 220 patients.

Trial Registration: ClinicalTrials.gov Identifier: NCT03029169, registered on 24.1.2017.

Keywords: Supraventricular arrhythmia, septic shock, propafenone, amiodarone, intensive care.

\section{Background}

The priorities in therapy of arrhythmias in sepsis and septic shock are prevention of diastolic heart failure, post-tachycardic heart dilatation and systolic heart failure with subsequent microtrombi formation within the heart and systemic embolisations. [1]. The classic stratification of diastolic dysfunction relates to patient's prognosis in septic shock [2]. A left ventricular relaxation disorder and a pseudonormal LV filling 
are more dependent on atrial filling compared to the restrictive LV filling which is often accompanied by a dilated poorly contracting left atrium.

The tendency to rather provide rate control than rhythm control therapy stems from the observed recurrence of arrhythmias and side effects of antiarrhythmics. Recent study on perioperative AF included the same antiarrhythmic agents and showed similar rates of electric cardioversion in $25 \%$ of the patients included either to a rhythm control or a rate control arm demonstrating the significant overlap between both approaches [3]. Nevertheless, the rate control and the resignation to restore atrial contribution to the ventricular filling contrasts with data showing that the loss of the atrial kick associates with two to five times increased mortality among critically ill patients [4-6].

In contrast to an acute onset arrhythmia an evidence demonstrating that reverting back to sinus rhythm (SR) [7] improves outcome is missing [8]. The absence of relevant prospective controlled randomized critical care trials (PRCTs) and also the limited applicability of major cardiology trials [9-11] may explain a lack of evidence pertaining to the arrhythmia management in the critically ill. The conclusions of available PRCTs are difficult to extrapolate to newly appearing SV arrhythmias in the septic and septic shock patients due to inclusion of all degrees of diastolic dysfunction without an attempt to stratify the degree of dependence of ventricular filling on atrial systole [8]. Furthermore, with the exemption of the AFCHF trial [9] the authors included patients with normal LV systolic function as well as all degrees of the LV systolic dysfunction and associated left atrial remodelling which relates to the ability to maintain SR $[12,13]$.

Besides improving oxygenation, preload and electrolyte corrections, electric cardioversion (DCC, preferably biphasic) is indicated in unstable patients with no contraindications and is more feasible in combination with an antiarrhythmic agent due to high rates of an early relapse of atrial fibrillation [14].

The data on various antiarrhythmic medications in current literature shows some important limitations, particularly the absence of an echocardiographic protocol before deciding on treatment [1]. Some of the available studies lack any attempt to avoid potentially unfeasible medication in an unstable, critically ill patient. For example, a large pool (36\%) of patients in sepsis was medicated with calcium channel blockers which can help with rate control at the cost of reducing ventricular contractility and promotion of vasodilatation. These side effects may critically impact upon haemodynamic stability in a patient with left ventricular compromise and/or septic vasoplegia [15]. In the studies suggesting beneficial effects of betablockers [16-19] the haemodynamic monitoring did not include echocardiography and the comparisons to control patients were fraught with high mortality of the control group [17]. Several limitations have to be considered prior to beta-blocker administration in septic shock patients. These are in particular exclusion of the severe LV systolic dysfunction, valve and conduction disorders $[18,20]$. The same limitations regarding the absence of echocardiographic assessment of haemodynamics apply to the majority of the case series on arrhythmias in sepsis and septic shock patients [1, 8]. Along with poorly defined inclusion and exclusion criteria, it might have lead to misleading conclusions [21]. 
The mainstay of antiarrhythmic therapy[1] is represented by amiodarone which is preferred for its lower cardiodepressant side effect compared to other agents and electric cardioversion [22-25]. The adverse effects of amiodarone involve many organ systems. Amiodarone contains iodine and interferes with thyroid function [26][27]. Hypotension may occur due to amiodarone's vasodilatatory effects and QTC prolongation associates with the occurence of torsades-des-pointes type of ventricular tachycardia. Other important side effects include corneal microdeposits, hepatic dysfunction [28, 29], intersticial pneumonia and pulmonary fibrosis [30,31], skin discoloration and neuropathies [32, 33]. High rates of amiodarone use contrast with its multiorgan side effects and its application even in cardiology patients with normal LV systolic function [34,35] demonstrate poor compliance with current guidelines [36].

The use of $1 \mathrm{C}$ class antiarrhythmic drugs in the treatment of SV arrhythmias in the critically ill has not been properly evaluated. There are only a few case reports available describing serious adverse effects apparently related to their dose related cardiotoxicity $[21,37,38]$. The use of $1 \mathrm{C}$ agents has been discouraged by reports describing poor outcome during long term administration in the cardiology population [21]. Consequently, $1 \mathrm{C}$ class agents like propafenone and flecainide [39], are scarcely used in the critically ill. In contrast to flecainide and encainide, propafenone is derived from propandiolamine, which is a chemical compound of betablockers and acts on the rapid depolarizing phase (phase 0 ) and also, to a minimal extent, on beta-adrenergic receptors [40-42]. Compared to flecainide, propafenone also lacks any evidence of its relationship to mortality [43].

Our recent retrospective study $[44,45]$ suggests that propafenone might be a drug of choice for a supraventricular tachyarrhythmia in septic shock patients with normal to moderately reduced EF_LV. The benefits of propafenone were also demonstrated in patients where amiodarone was not capable of maintaining the SR. Another recent retrospective study found faster and more succesful cardioversion with propafenone as compared to amiodarone for new onset atrial fibrillation in an emergency department. The safety profiles of the two agents were not different [46]. The routine dosage of propafenone might be feasible to restore SR without an adverse effect on haemodynamics and with a possible benefit on the outcome (Fig.1) [44,45]. A chance to cardiovert seemed to be significantly higher under propafenone than in amiodarone and was close to the cardioversion rates of the betablocker metoprolol. No secondary arrhythmias or conduction disorders requiring treatment other than adjustment of the rate of infusion were observed $[44,45]$.

Administering propafenone for SV arrhythmia in septic shock associated with lower ICU and 28-day mortalities compared to amiodarone however, the difference did not reach statistic significance. A statistically significant 12-month mortality benefit in favour of propafenone was found (Fig.1). A typical patient benefiting from propafenone has no significant structural heart disease, i.e. typically has normal or mildly reduced left ventricular systolic function.

The authors hypothesize that actively pursuing sinus rhythm and cardioverting patients may contribute to the therapy of diastolic dysfunction with a positive impact on mortality [8]. It seems that the degree of left ventricular dependence on atrial kick would be an important entity when deciding between rhythm and 
rate control in SV arrhythmia in septic critically ill patients. The rate control modality should be reserved for a chronic AF and in situations when sinus rhythm is difficult to maintain due to high dosage of vasoactive agents. The current trial is intended to prospectively verify the efficacy and safety of propafenone administered under echocardiography control in the critically ill with septic shock. The trial also challenges the concept of amiodarone applied as a relatively toxic universal antiarrhythmic agent with a similar short-term safety profile as propafenone, whilst being slower and less efficient in cardioverting a supraventricular arrhythmia in a septic shock patient.

\section{Methods}

We designed a prospective double blinded randomized trial comparing propafenone to amiodarone administered for a SV arrhythmia in critically ill patients with septic shock.

\section{Primary aims}

The trial should prove that propafenone is more efficient than amiodarone in cardioverting a SV arrhythmia in patients with normal to moderately reduced EF_LV at $24 \mathrm{~h}$ from the onset. The rationale stems from the retrospective data set where the primary cardioversion rate of SV arrhythmia under propafenone was $88.9 \%$ versus $73.5 \%$ under amiodarone $[44,45]$. The authors also expect faster cardioversion under propafenone and lower rates of arrhythmia recurrence in the propafenone group. Despite prejudices arising particularly from the CAST trial and case reports on dose dependent toxicity, research should prove the safety of the $1 \mathrm{C}$ class agent propafenone given within the summary of product characteristics $[37,39]$. The retrospective study $[44,45]$ has shown that the ICU and 28-day mortalities of patients treated with propafenone were better than the parameters of the amiodarone patients ( $30.4 \%$ vs $40.4 \%$ and $39.5 \%$ vs $49.6 \%$, respectively). The differences did not reach statistic significance however, the propafenone administration did not increase mortality as suggested by the older trials on non-ICU patients $[21,37,38]$. If proven, the physicians could avoid a widespread use of amiodarone in the critically ill. The use of amiodarone conveys side effects and multiorgan toxicity [27, 41]. Moreover, patients with a supraventricular arrhythmia treated with propafenone had an adjusted 12months survival similar to patients medicated with the betablocker metoprolol and both groups had significantly better 12-month survival than the critically ill treated with amiodarone (Fig.1).

The cardioverted patients (rhythm control) may showcase better outcome parameters (ICU mortality, 28day mortality, 1-year mortality) than those remaining in an acute onset arrhythmia (rate control). A rationale beyond this hypothesis is in the pilot study $[44,45]$ which also included patients with severe LV dysfunction and associated higher rates of recurrent SV arrhythmias. Focusing on only normal to moderate LV systolic dysfunction may minimize bias associated with arrhythmia treatment of patients with severe LV systolic dysfunction. Likewise, those patients were included in the published trials dealing with either $1 \mathrm{C}$ class antiarrhythmics (e.g. CAST trial,[21]) or in the trials studying rhythm vs rate control (e.g. AFFIRM, RACE or AF-CHF Trial,[3, 10, 47, 48]). The retrospective data on 234 patients in septic shock with SV arrhythmia $[44,45]$ showed a long-term benefit of rhythm control which was not statistically 
significant. Due to high success of rhythm control therapy ( $74.4 \%$ and $87 \%$ excluding chronic AF) the group with persisting acute onset SV arrhythmia was significantly smaller in number causing asymmetry in statistic evaluation. This may account for not signicant difference between the outcome of the cardioverted versus those remaining in the SV arrhythmias (Fig.2).

\section{Secondary aims}

The presence of a transmitral diastolic A wave and its higher velocity-time integral (VTI) at $4 \mathrm{~h}$ post cardioversion would indicate a presence of mechanical sinus rhythm. The incidence of a small or negligible A wave or only the presence of electric sinus in the absence of its mechanical correlate could be related to the indexed left atrial end-systolic volume (LAVi) and to a recurrence of a SV arrhythmia[49, 50].

The LAVi in all patients and altered filling pressures estimated by echocardiography could be predictive of recurrent arrhythmia $[12,51]$.

Propafenone would be more efficient than amiodarone in patients with pulmonary hypertension and RV dysfunction without left ventricular systolic dysfunction.

Approximately $8-25 \%$ of the critically ill suffer from SV arrhythmias [4-6, 44, 45] and an echocardiography driven prediction of cardioversion has not been explored in the critically ill patients in the available literature. Hence, a complex echo assessment may contribute to the decision whether to aim for rhythm or for rate control only. An application of simple 2D echo parameters like biplanar endsystolic LAVi or just endsystolic LA size may be included in the focused critical care echocardiography performed by an intensivist on a patient with arrhythmia of unknown duration. The evaluation of the doppler parameters will depend on rhythm, heart rate, regularity of arrhythmia and peripheral pulse deficit $[49,51]$.

\section{Flow chart (Fig.3, Fig.4) and study setting}

Patients are randomized by the unblinded team lead by a research nurse. The planned number of included patients is 100 in each arm of the study with a total of 220 randomized patients. A dropout of $10 \%$ is anticipated. The estimated duration of the study is 4 years including follow up. The patients have been recruited since November 2017 in three university hospital ICUs. The department of Anaesthesia and Intensive Care of the General University Hospital has been performing for years as a teaching centre for critical care echocardiography and ultrasound. Together with the Coronary Care Unit of the General University Hospital both departments are integrated as a Complex Cardiovascular Centre. The department of Anaesthesia and Intensive Care of the University Hospital Kralovské Vinohrady is a mainstay of the Complex Prague Traumacentre.

\section{Inclusion Criteria}

The study targets patients in septic shock with a new onset SV arrhythmia or known paroxysmal SV arrhythmia who show normal or mildly to moderately reduced LV systolic function according to the 
echocardiography examination (i.e. EF_LV $>/=35 \%$ ). A diagnosis of septic shock is made according to the 2016 definition[52] as sepsis with a vasopressor requirement to maintain a mean arterial pressure of 65 $\mathrm{mm} \mathrm{Hg}$ or greater. The arterial lactate level should be greater than $2 \mathrm{mmol} / \mathrm{L}$ in the absence of hypovolemia or low cardiac output. The highest arterial lactate level is recorded, i.e. lactate $<2.0 \mathrm{mmol} / \mathrm{l}$ at the time of randomization does not exclude a patient from the study. This might also be justified by the reported incidence of the sepsis related cardiac dysfunction which is highest 72-96h after an onset of septic shock [53]. The presence of a suspected infection is for the purpose of this study defined as a positivity of at least one inflammatory marker of the monitored CRP and PCT and a clinical decision to administer antibiotic treatment for a specified infection source.

\section{Exclusion Criteria}

The study respects all exclusion criteria for a blinded administration of propafenone or amiodarone. These are in particular severe LV systolic dysfunction (i.e. $\mathrm{EF}<35 \%$ ), a history indicating more than $1^{\text {st }}$ degree AV block and a high dose vasopressor therapy represented by continuous noradrenaline administration of more than $1.0 \mathrm{ug} / \mathrm{kg}$. min. Contraindications to randomisation are known intolerance to amiodarone or propafenone, iodine allergy and an active thyroid disease other than chronic hormone substitution for benign goiter. Chronic persistant AF represents an exclusion while known chronic paroxysmal AF is not an exclusion criterion. Patients dependent on a pacemaker or after a Maze procedure are also excluded.

\section{Interventions and research protocol}

Patients in septic shock with a new onset supraventricular arrhythmia will have a haemodynamic examination provided according to the study protocol. With the onset of arrhythmia, the usual treatment is expected including preload correction, reduction of unnecessary vasopressors, ion supplementation (aiming particularly for $\mathrm{K}^{+}>4.0 \mathrm{mM}$ and $\mathrm{Mg}^{2+}>1.0 \mathrm{mM}$ ) and maintenance of tissue oxygen delivery. Echocardiography should also guide optimization of preload.

The complex protocol is formatted in an electronic case report form (CRF). After checking up the inclusion and exclusion criteria the CRF allocates the patients randomly using built in software (www.randomization.com) into the propafenone or amiodarone arm.

The patient's characteristics include the illness severity scores, source of septic shock, data on mechanical ventilation and homeostasis, baseline haemodynamic data, baseline laboratory data, patient 's medications, haemodynamic data at proposed steps plus follow up data including outcome.

Haemodynamic evaluation includes ICU standard plus echocardiography. The study team involves 8 intensivists with an European Accreditation in Echocardiography (either ESC or EACTA backed) and three qualified cardiologists-intensivists. 
By no means is an antiarrhythmic given out of the summary of product characteristics. Both arms will have standard treatment, there are no limits to indicate electric cardioversion as part of treatment. Electric cardioversion is indicated anytime in haemodynamic compromise and in signs of low cardiac output and/or loss of perfusion pressures due to arrhythmia. It will also be indicated should there be observed not satisfactory effect of the antiarrhythmic medication during the first $24 \mathrm{~h}$ from the start of arrhythmia.

The propafenone arm constitutes administering a bolus of $35-70 \mathrm{mg}$ of intravenous propafenone followed by a continuous infusion of $400-840 \mathrm{mg} / 24 \mathrm{~h}$ in a black syringe. The amiodarone arm constitutes administering a bolus of $150-300 \mathrm{mg}$ of intravenous amiodarone followed by a continuous infusion of $600-1800 \mathrm{mg} / 24 \mathrm{~h}$ in a black syringe.

A 12-lead ECG is taken every $12 \mathrm{~h}$ whilst the antiarrhythmic infusion. Besides echocardiography prerandomization the control echocardiography is performed $1 \mathrm{~h}$ post cardioversionand $4 \mathrm{~h}$ post cardioversion. Echocardiography is performed also every day until cardioversion, it is also mandatory in any kind of haemodynamic instability. All the Doppler measurements are recorded at end-expiration and 3 cardiac cycles, when sinus rhythm, and 5-10, during arrhythmia, are analysed and averaged. All recordings should be acquired with an ECG (lead II), and ideally, at the speed of $100 \mathrm{~mm} / \mathrm{s}$.

Electric cardioversion is allowed anytime when urgently indicated and even when arrhythmia persists while on the antiarrhythmic medication. If electrically cardioverted in addition to administered pharmacotherapy, then echocardiography is performed $1 \mathrm{~h}$ post cardioversion and $4 \mathrm{~h}$ post cardioversion.

If cardioverted later than until $24 \mathrm{~h}$ then echocardiographies are performed at $1 \mathrm{~h}$ and $4 \mathrm{~h}$ after cardioversion, the times of cardioversion and arrhythmia relapses are always recorded.

\section{Primary outcome measures}

1. The efficacy in restoration of sinus rhythm assessed as the proportion of patients who are in sinus rhythm 24 hours after the beginning of the infusion of the study drug and remain in sinus rhythm until discharge from ICU. Primary outcome will be assessed in all randomised patients (i.e. intention to treat analysis).

2. A-priori defined subgroup analysis: Primary outcome will be analysed in the following subgroups of patients:

3. with and without indexed left atrial endsystolic volume (LAVi) higher than $>40 \mathrm{ml} / \mathrm{m}^{2}$.

4. with and without pulmonary hypertension (defined as PAPs $>40 \mathrm{mmHg}$ ) associated with moderate to severe RV dysfunction (dilated RV with TAPSE $<15 \mathrm{~mm}$ )

\section{Secondary outcome measures}

1. The cumulative proportion of patients receiving rescue treatment for arrhythmia defined as direct current cardioversion or an alternative antiarrhythmic drug during the first 24 hours (cross-over from one arm to the other resulting in unblinding of the study, e.g. from amiodarone to propafenone due to 
a persisting arrhythmia or from propafenone to amiodarone due to a decrease in LV systolic function).

2. The cumulative proportion of patients receiving rescue treatment for arrhythmia defined as direct current cardioversion, cross-over to the alternative study drug or other antiarrhythmic drug during ICU stay.

3. Mortality at discharge from ICU, at 28 days and at 1 year.

4. Vasopressor-free days at day 28.

\section{Safety issues and patient's monitoring}

TTE is performed at the onset of the arrhythmia and daily until cardioversion, also at $1 \mathrm{~h}$ and at $4 \mathrm{~h}$ after cardioversion. TTE is also acquired in any kind of haemodynamic instability (i.e. change in vasopressor support). This is important to avoid administering a potentialy cardiodepressant propafenone in a patient developing septic cardiomyopathy. 12 hourly 12-lead ECG for monitoring of conduction times (PQ, QRS, QTC) is performed while the patient is on the antiarrhythmic infusion. In case of an AV block of the first degree or extension of the conduction times (QRS or QTC) the slowing or temporary ceasing of the medication in relation to heart rate is mandatory. Adjustment of the infusion rate or eventual termination of an antiarrhythmic medication does not exclude the patient from the study. Ceasing of medication after reaching sinus rhythm does not exclude the patient either. If an infusion is interrupted and restarted then the number of infusion hours are counted up as a sum of infusion hours.

In case of a progression of septic cardiomyopathy and a decrease of contractility (decrease of EFLV to $<35 \%$ ) or a progression of mitral regurgitation with a risk of low cardiac output the study drug is unblinded and propafenone discontinued. Further treatment is decided by the clinician. If the study is unblinded due to haemodynamic instability, the second drug after study arm cross-over is administered without an initial bolus.

Anytime the patient becomes haemodynamically unstable or has another reason (as per discretion of the treating clinician) to benefit from electric cardioversion (DCC), then DCC is delivered without delay.

Should there be a concern at any point in time about the safety of the drug, the treating clinicians are encouraged to unblind the treatment drug without delay, and alter the treatment accordingly. The course of the trial is regularly reported to the hospital Ethical Board which acts as the research supervising body. The minimum frequency of the report is once per year throughout the duration of the trial which is proposed from 2018 till 2021.

\section{Statistics and power analysis}

The logistic regression and time-to-event (Cox) regression with and without adjustment for baseline patients' characteristics will be applied in the statistic analysis. The multivariate analysis will include the 
patients' baseline parameters which would be correlated with an analysed outcome parameter and will be inhomogenously distributed within the study groups regardless of the randomisation. The required number of patients is based on the power analysis and data from the pilot retrospective study $[44,45]$.

The entry parameters for the sample size analysis were estimated by the probabilities of cardioversion of $75 \%$ for the amiodarone group and $90 \%$ for the propafenone group within $24 \mathrm{~h}$ from the onset of arrhythmia, randomisation ratio $1: 1, p=0.05$ and power 0.8 . To achieve a statistically significant difference under these conditions 100 patients need to be included into each group, altogether 200 patients into the trial. Assuming 10\% drop out the authors plan to randomize 220 patients.

\section{Discussion}

The available literature on SV arrhythmias in septic shock shows critically ill patients in septic shock with a high predicted mortality, IPPV rate of $99 \%$ and high rates of CRRT $(27-31 \%)[44,45]$. Up to now all the authors adhered to the septic shock criteria based on volume non-responsive SIRS with a need for a vasopressor and antibiotic therapy administered for an infectious source [54]. Applying the novel septic shock criteria of 2016 [52] may increase specificity at the cost of lacking sensitivity to include even those who could potentially benefit from septic shock therapy [55]. In addition, certain studies show high inflammatory markers (e.g. PCT) suggesting a high rate of bacteraemia among patients categorized according to the older SIRS criteria. If applying the results of the current trial to less severe patients, e.g. those classified according to the older criteria, the SOFA score and a median arterial lactate level may serve as controls adjusting studied population in context of the novel septic shock criteria published in 2016 [52].

The parallel application of the ECG and echocardiography in the arrhythmia patients allows to indicate antiarrhythmics with the exclusion of a more cardiodepressant medication (propafenone) in severe LV dysfunction and also to correct preload when attempting for cardioversion. Echocardiography helps also to decide whether to cardiovert a patient with unknown history. A finding of a significantly dilated left atrium or valvular disorder may associate with chronic AF.

The hypothesis that propafenone might be superior to amiodarone in cardioverting newly appearing SV arrhythmia with an impact on the long term outcome may not be proved due to the confounding factors of the retrospective study [44,45]. Albeit being statistically insignificant, the LV systolic function was higher in propafenone and betablocker patients compared to those on amiodarone. The dosage of noradrenaline was significantly higher in the amiodarone group likely reflecting the severity of septic shock. In comparison to propafenone the higher illness severity expected in the amiodarone group was not found to associate with any other available strong outcome predictor like rates of CRRT, procalcitonin, SOFA and APACHE II and the dosage of noradrenaline was not related to the 12-month mortality in the multivariate regression $[44,45]$. The majority of severe LV systolic dysfunctions were medicated with amiodarone as well as patients on a higher dosage of noradrenaline compared to the patients with 
moderate to mild LV systolic dysfunction and the lower dosage of noradrenaline in the propafenone and betablocker groups $[44,45]$.

The retrospective study included also patients with a cross-over from a not successful antiarrhythmic therapy to another group during 24 hours as part of the rhythm control strategy. This increased the pool of the propafenone patients from $17.5 \%$ where it was used as a primary antiarrythmic to $33 \%$ after administering the agent in patients who were not able to cardiovert and maintain sinus rhythm on amiodarone $[44,45]$. This might represent a so far not reported synergistic effect of the two antiarrhythmic agents on achieving a high cardioversion rate, yet with a very acceptable safety profile [44, 45]. The current prospective trial allows a cross-over between the arms however, only in a haemodynamic instability and with immediate unblinding.

The observed median age in an adult ICU varies around 55-65 years. The age related prevalence of hypertension and ischaemic heart disease suggests a large proportion of patients with a benefit of atrial systole and thus an indication for the rhythm control approach [8]. The prevalence of newly occuring SV arrhythmias and the broad spectrum of potentially reversible triggers in the critically ill offer an opportunity for cardioversion in closely monitored patients rather than in ambulatory patients in cardiology. Moreover, a critical illness like septic shock is often fraught with diastolic heart compromise and to restore sinus rhythm might be of paramout importance for the therapy of diastolic heart failure.

\section{Abbreviations}

AF atrial fibrillation, APACHE II acute physiologic and chronic health evaluation, AV atrio-ventricular, CRP C-reactive protein, CRRT continuous renal replacement therapy, DCC direct current cardioversion, D02/VO2 oxygen delivery/oxygen consumption, EF ejection fraction, EF_LV ejection fraction of left ventricle, ICU intensive care unit, $\mathrm{K}+$ plasmatic potassium, LA left atrium, LAVi indexed end-systolic left atrial volume, LV left ventricle/ left ventricular, LVOT left ventricular outflow tract, Mg2+ plasmatic magnesium, PAPs pulmonary artery systolic pressure, PCT procalcitonin, PRCT prospective controlled randomized trial, RV right ventricle, SIRS systemic inflammatory response syndrome, SOFA sequential organ function assessment, SR sinus rhythm, SV supraventricular, TAPSE tricuspid annular plane excursion, TTE transthoracic echocardiography, VTI velocity-time integral

\section{Declarations}

\section{Trial status:}

The trial is recruiting patients according to its $2^{\text {nd }}$ protocol version approved by the Ethical Board on the $6^{\text {th }}$ October 2017. The recruitment has begun on the $23^{\text {rd }}$ October 2017 and is expected to be completed in December 2021.

\section{Ethics approval and consent to participate:}


The local ethical approval has been confirmed from the Ethics Committee of the $1^{\text {st }}$ Medical Faculty and General University Hospital (ref approval no. 1691/16 S-IV) and from the Ethics Committee of the $3^{\text {rd }}$ Medical Faculty and University Hospital Kralovske Vinohrady. We will not begin recruiting at other centres in the trial until local ethical approval has been obtained.

\section{Consent for publication:}

Not Applicable

\section{Availability of data and materials: T}

he study repository will be created with the dataset available after study completion. At present the trial recruits patients through the electronic case report form at https://ecrf.fnkv.cz:9090/apex/f? $p=115: 101: 14397031770294:: N O$ :

\section{Competing interests:}

None

\section{Funding:}

The protocol has received a four year (2018-2022) grant support from the Czech Health Research Council, AZV No. NV18-06-00417, commencing on the $1^{\text {st }}$ of May 2018.

Financial disclosure statement: The echocardiographic devices used for the study purpose were financed from project reg.no. CZ.2.16/3.1.00/21565 from OP Prague Competitiveness. This sponsor did not have any role in study design, collection, analysis and interpretation of the data, in the writing of the paper and in the decision to submit the article for publication.

\section{Author's Contributions:}

$\mathrm{MB}$ - study coordinator, concept and design, drafting, revisions and approval of articles, provision of funding. PW, FD - concept and design, electronic case report form, statistics, article revisions, data collection. MP, JR, MO, VM, MM, TB, RS, JP, PB, ES, MF, ZS, MS - data collection, article revisions. OS article revisions, data collection, unblinded team coordination.

\section{Acknowledgements:}

Not Applicable

\section{References}

1. Balik M, Matousek V, Maly M, Brozek T: Management of arrhythmia in sepsis and septic shock. Anaesthesiology intensive therapy 2017, 49(5):419-429. 
2. Poelaert J, Declerck C, Vogelaers D, Colardyn F, Visser CA: Left ventricular systolic and diastolic function in septic shock. Intensive care medicine 1997, 23(5):553-560.

3. Gillinov AM, Bagiella E, Moskowitz AJ, Raiten JM, Groh MA, Bowdish ME, Ailawadi G, Kirkwood KA, Perrault LP, Parides MK et al: Rate Control versus Rhythm Control for Atrial Fibrillation after Cardiac Surgery. The New England journal of medicine 2016, 374(20):1911-1921.

4. Klein Klouwenberg PM FJ, Kuipers S, Ong DS, Peelen LM, van Vught LA, Schultz MJ, van der Poll T, Bonten MJ, Cremer OL; MARS consortium.: Incidence, Predictors and Outcomes of New-onset Atrial Fibrillation in Critically III Patients with Sepsis: a Cohort Study. Am J Respir Crit Care Med 2016.

5. Kuipers S KKP, Cremer OL: Incidence, risk factors and outcomes of new-onset atrial fibrillation in patients with sepsis: a systematic review. Crit Care 2014, 18(6):688.

6. Arrigo M, Bettex D, Rudiger A: Management of atrial fibrillation in critically ill patients. Critical care research and practice 2014, 2014:840615.

7. Liu WC, Lin WY, Lin CS, Huang HB, Lin TC, Cheng SM, Yang SP, Lin JC, Lin WS: Prognostic impact of restored sinus rhythm in patients with sepsis and new-onset atrial fibrillation. Crit Care 2016, 20(1):373.

8. Balik M: New-onset atrial fibrillation in critically ill patients - Implications for rhythm rather than rate control therapy? International journal of cardiology 2018, 266:147-148.

9. Roy D, Talajic M, Nattel S, Wyse DG, Dorian P, Lee KL, Bourassa MG, Arnold JM, Buxton AE, Camm AJ et al: Rhythm control versus rate control for atrial fibrillation and heart failure. The New England journal of medicine 2008, 358(25):2667-2677.

10. Van Gelder IC, Hagens VE, Bosker HA, Kingma JH, Kamp O, Kingma T, Said SA, Darmanata JI, Timmermans AJ, Tijssen JG et al: A comparison of rate control and rhythm control in patients with recurrent persistent atrial fibrillation. The New England journal of medicine 2002, 347(23):1834-1840.

11. Investigators. AFADS: Maintenance of sinus rhythm in patients with atrial fibrillation: an AFFIRM substudy of the first antiarrhythmic drug. Journal of the American College of Cardiology 2003, 42(1):20-29.

12. Marchese P, Bursi F, Delle Donne G, Malavasi V, Casali E, Barbieri A, Melandri F, Modena MG: Indexed left atrial volume predicts the recurrence of non-valvular atrial fibrillation after successful cardioversion. European journal of echocardiography : the journal of the Working Group on Echocardiography of the European Society of Cardiology 2011, 12(3):214-221.

13. Fornengo C, Antolini M, Frea S, Gallo C, Grosso Marra W, Morello M, Gaita F: Prediction of atrial fibrillation recurrence after cardioversion in patients with left-atrial dilation. Eur Heart $J$ Cardiovasc Imaging 2015, 16(3):335-341.

14. Arrigo M, Jaeger N, Seifert B, Spahn DR, Bettex D, Rudiger A: Disappointing Success of Electrical Cardioversion for New-Onset Atrial Fibrillation in Cardiosurgical ICU Patients. Critical care medicine 2015, 43(11):2354-2359.

15. Walkey AJ, Evans SR, Winter MR, Benjamin EJ: Practice Patterns and Outcomes of Treatments for Atrial Fibrillation During Sepsis: A Propensity-Matched Cohort Study. Chest 2016, 149(1):74-83. 
16. Morelli A DA, Ertmer C, Rehberg S, Kampmeier T, Orecchioni A, D'Egidio A, Cecchini V, Landoni G, Pietropaoli P, Westphal M, Venditti M, Mebazaa A, Singer M.: Microvascular effects of heart rate control with esmolol in patients with septic shock: a pilot study. Crit Care Med 2013, 41(9):21622168.

17. Morelli A EC, Westphal M, Rehberg S, Kampmeier T, Ligges S, Orecchioni A, D'Egidio A, D'Ippoliti F, Raffone C, Venditti M, Guarracino F, Girardis M, Tritapepe L, Pietropaoli P, Mebazaa A, Singer M.: Effect of heart rate control with esmolol on hemodynamic and clinical outcomes in patients with septic shock: a randomized clinical trial. JAMA 2013, 310(16):1683-1691.

18. Balik M RJ, Leden P, Zakharchenko M, Otahal M, Bartakova H, Korinek J: Concomitant use of beta-1 adrenoreceptor blocker and norepinephrine in patients with septic shock. Wien Klin Wochenschr 2012, 124:552-556.

19. Balik M RJ, Leden P, Zakharchenko M, Otahal M, Bartakova H, Korinek J: Concomitant use of beta-1 adrenoreceptor blocker and norepinephrine in patients with septic shock. Reply to a letter to the authors. Wien Klin Wochenschr 2014, 126(7-8):246-247.

20. McLean AS TF, Vieillard-Baron A: Beta-blockers in septic shock to optimize hemodynamics? No. Intensive Care Med 2016.

21. Echt DS LP, Mitchell LB, Peters RW, Obias-Manno D, Barker AH, Arensberg D, Baker A, Friedman L, Greene $\mathrm{HL}$, et al.: Mortality and morbidity in patients receiving encainide, flecainide, or placebo. The Cardiac Arrhythmia Suppression Trial. NEJM 1991, 324(12):781-788.

22. Arrigo M BD, Rudiger A: Management of atrial fibrillation in critically ill patients. Crit Care Res Pract 2014, 2014(840615).

23. Kirchhof P AB, Darius H, De Caterina R, Le Heuzey JY, Schilling RJ, Schmitt J, Zamorano JL.: Management of atrial fibrillation in seven European countries after the publication of the 2010 ESC Guidelines on atrial fibrillation: primary results of the PREvention oF thromboemolic eventsEuropean Registry in Atrial Fibrillation (PREFER in AF). Europace 2014, 16(1):6-14.

24. Sleeswijk ME VNT, Tulleken JE, Ligtenberg JJ, Girbes AR, Zijlstra JG: Clinical review: treatment of new-onset atrial fibrillation in medical intensive care patients - a clinical framework. Crit Care 2007, 11(6):233.

25. Arrigo M JN, Seifert B, Spahn DR, Bettex D, Rudiger A.: Disappointing Success of Electrical Cardioversion for New-Onset Atrial Fibrillation in Cardiosurgical ICU Patients. Crit Care Med 2015, 43(11):2354-2359.

26. Hassan S, Ayoub W, Hassan M, Wisgerhof M: Amiodarone-induced myxoedema coma. BMJ case reports 2014, 2014.

27. Hofmann A NC, Ofluoglu S, Holzmannhofer J, Strohmer B, Pirich C: Incidence and predictability of amiodarone-induced thyrotoxicosis and hypothyroidism. Wien Klin Wochenschr 2008, 120(1516):493-498.

28. Jaiswal P, Attar BM, Yap JE, Devani K, Jaiswal R, Wang Y, Szynkarek R, Patel D, Demetria M: Acute liver failure with amiodarone infusion: A case report and systematic review. Journal of clinical 
pharmacy and therapeutics 2017.

29. Ratz Bravo AE, Drewe J, Schlienger RG, Krahenbuhl S, Pargger H, Ummenhofer W: Hepatotoxicity during rapid intravenous loading with amiodarone: Description of three cases and review of the literature. Critical care medicine 2005, 33(1):128-134; discussion 245-126.

30. Charles PE, Doise JM, Quenot JP, Muller G, Aube H, Baudouin N, Piard F, Besancenot JF, Blettery B: Amiodarone-related acute respiratory distress syndrome following sudden withdrawal of steroids. Respiration; international review of thoracic diseases 2006, 73(2):248-249.

31. Singh VK, Maheshwari V: Acute Respiratory Distress Syndrome Complicated by Amiodarone Induced Pulmonary Fibrosis: Don't Let Your Guard Down. Journal of clinical and diagnostic research : JCDR 2017, 11(4):Ud01-ud02.

32. Hughes $M$, Binning $A$ : Intravenous amiodarone in intensive care. Time for a reappraisal? Intensive care medicine 2000, 26(12):1730-1739.

33. Papiris SA, Triantafillidou C, Kolilekas L, Markoulaki D, Manali ED: Amiodarone: review of pulmonary effects and toxicity. Drug safety 2010, 33(7):539-558.

34. Allen LaPointe NM, Dai D, Thomas L, Piccini JP, Peterson ED, Al-Khatib SM: Antiarrhythmic drug use in patients $<65$ years with atrial fibrillation and without structural heart disease. The American journal of cardiology 2015, 115(3):316-322.

35. Gwag HB, Chun KJ, Hwang JK, Park SJ, Kim JS, Park KM, On YK: Which antiarrhythmic drug to choose after electrical cardioversion: A study on non-valvular atrial fibrillation patients. PloS one 2018, 13(5):e0197352.

36. Kirchhof P, Benussi S, Kotecha D, Ahlsson A, Atar D, Casadei B, Castella M, Diener HC, Heidbuchel H, Hendriks $\mathrm{J}$ et al: 2016 ESC Guidelines for the management of atrial fibrillation developed in collaboration with EACTS. European heart journa/ 2016, 37(38):2893-2962.

37. Chevalier P D-DA, Burri H, Cucherat M, Kirkorian G, Touboul P.: Amiodarone versus placebo and class Ic drugs for cardioversion of recent-onset atrial fibrillation: a meta-analysis. Journal of the American College of Cardiology 2003, 41(2):255-262.

38. Courand PY SF, Ranc S, Mullier A, Kirkorian G, Bonnefoy E.: Arrhythmogenic effect of flecainide toxicity. Cardiology Journal 2013, 20(2):203-205.

39. Aliot E CA, Crijns HJ, Goette A, Tamargo J: Twenty-five years in the making: flecainide is safe and effective for the management of atrial fibrillation. Europace 2011, 13(2):161-173.

40. Varon J, Marik PE: Irwin and Rippe's intensive care medicine. In., 6th edn. Edited by Irwin RS, Rippe JM. Philadelphia: Wolters Kluwer Health/Lippincott Williams \& Wilkins; 2008: 1855-1869.

41. Ganetsky M BE: Antiarythmic agents. In: Intensive care medicine. 6th edn. Edited by Irwin RS RJ. Philadelphia: Wolters Kluwer/Lippincott, Williams\&Wilkins; 2008: 1486-1498.

42. Stoschitzky K, Stoschitzky G, Lercher P, Brussee H, Lamprecht G, Lindner W: Propafenone shows class Ic and class II antiarrhythmic effects. Europace 2016, 18(4):568-571. 
43. Lafuente-Lafuente C, Valembois L, Bergmann JF, Belmin J: Antiarrhythmics for maintaining sinus rhythm after cardioversion of atrial fibrillation. The Cochrane database of systematic reviews 2015(3):Cd005049.

44. Balik M, Kolnikova I, Maly M, Waldauf P, Tavazzi G, Kristof J: Propafenone for supraventricular arrhythmias in septic shock-Comparison to amiodarone and metoprolol. Journal of critical care 2017, 41:16-23.

45. Balik M, Maly M, Brozek T, Brestovansky P: Propafenone for supraventricular arrhythmias in septic shock - Comparison to amiodarone and metoprolol. The author's reply. Journal of critical care 2018, 45:247-248.

46. Bonora A, Turcato G, Franchi E, Taioli G, Dilda A, Zerman G, Maccagnani A, Pistorelli C, Olivieri O: Efficacy and safety in pharmacological cardioversion of recent-onset atrial fibrillation: a propensity score matching to compare amiodarone vs class IC antiarrhythmic drugs. Internal and emergency medicine 2017, 12(6):853-859.

47. Wyse DG, Waldo AL, DiMarco JP, Domanski MJ, Rosenberg Y, Schron EB, Kellen JC, Greene HL, Mickel MC, Dalquist JE et al: A comparison of rate control and rhythm control in patients with atrial fibrillation. The New England journal of medicine 2002, 347(23):1825-1833.

48. ARISE Investigators; ANZICS Clinical Trials Group PS, Delaney A, Bailey M, Bellomo R, Cameron PA, Cooper DJ, Higgins AM, Holdgate A, Howe BD, Webb SA, Williams P.: Goal-directed resuscitation for patients with early septic shock. NEJM 2014, 371(16):1496-1506.

49. Chung $C S$, Kovacs $S J$ : Consequences of increasing heart rate on deceleration time, the velocity-time integral, and E/A. The American journal of cardiology 2006, 97(1):130-136.

50. Fornengo C AM, Frea S, Gallo C, Grosso Marra W, Morello M, Gaita F.: Prediction of atrial fibrillation recurrence after cardioversion in patients with left-atrial dilation. Eur Heart J Cardiovasc Imaging 2015, 16(3):335-341.

51. Nagueh SF AC, Gillebert TC, Marino PN, Oh JK, Smiseth OA, Waggoner AD, Flachskampf FA, Pellikka PA, Evangelisa A.: Recommendations for the evaluation of left ventricular diastolic function by echocardiography. European journal of echocardiography : the journal of the Working Group on Echocardiography of the European Society of Cardiology 2009, 10(2):165-193.

52. Singer M DC, Seymour CW, Shankar-Hari M, Annane D, Bauer M, Bellomo R, Bernard GR, Chiche JD, Coopersmith CM, Hotchkiss RS, Levy MM, Marshall JC, Martin GS, Opal SM, Rubenfeld GD, van der Poll T, Vincent JL, Angus DC: The Third International Consensus Definitions for Sepsis and Septic Shock (Sepsis-3). JAMA 2016, 315(8):801-810.

53. Repesse $X$, Charron C, Vieillard-Baron A: Evaluation of left ventricular systolic function revisited in septic shock. Crit Care 2013, 17(4):164.

54. Levy MM, Fink MP, Marshall JC, Abraham E, Angus D, Cook D, Cohen J, Opal SM, Vincent JL, Ramsay G: 2001 SCCM/ESICM/ACCP/ATS/SIS International Sepsis Definitions Conference. Crit Care Med 2003, 31(4):1250-1256. 
55. Sterling SA, Puskarich MA, Glass AF, Guirgis F, Jones AE: The Impact of the Sepsis-3 Septic Shock Definition on Previously Defined Septic Shock Patients. Critical care medicine 2017, 45(9):14361442.

\section{Figures}

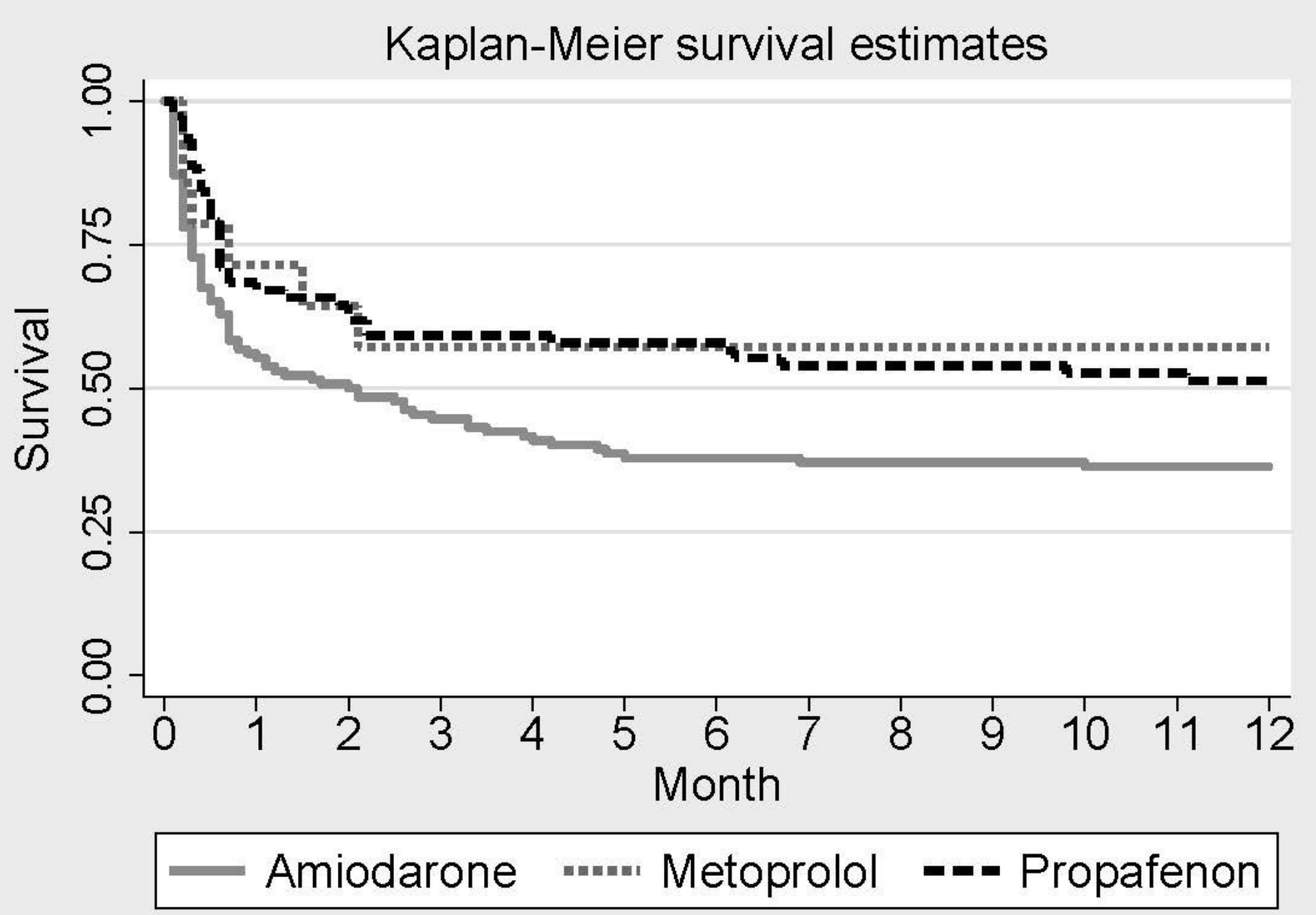

Figure 1

Univariate analysis showing long term survival of the propafenon patients similar to the metoprolol group and higher than in the amiodarone medicated patients in septic shock (HR1.76(1.06; 2.3), $p=0.024)$. Copied from the author's pilot retrospective study [44] . 


\section{Adjusted survival estimates}

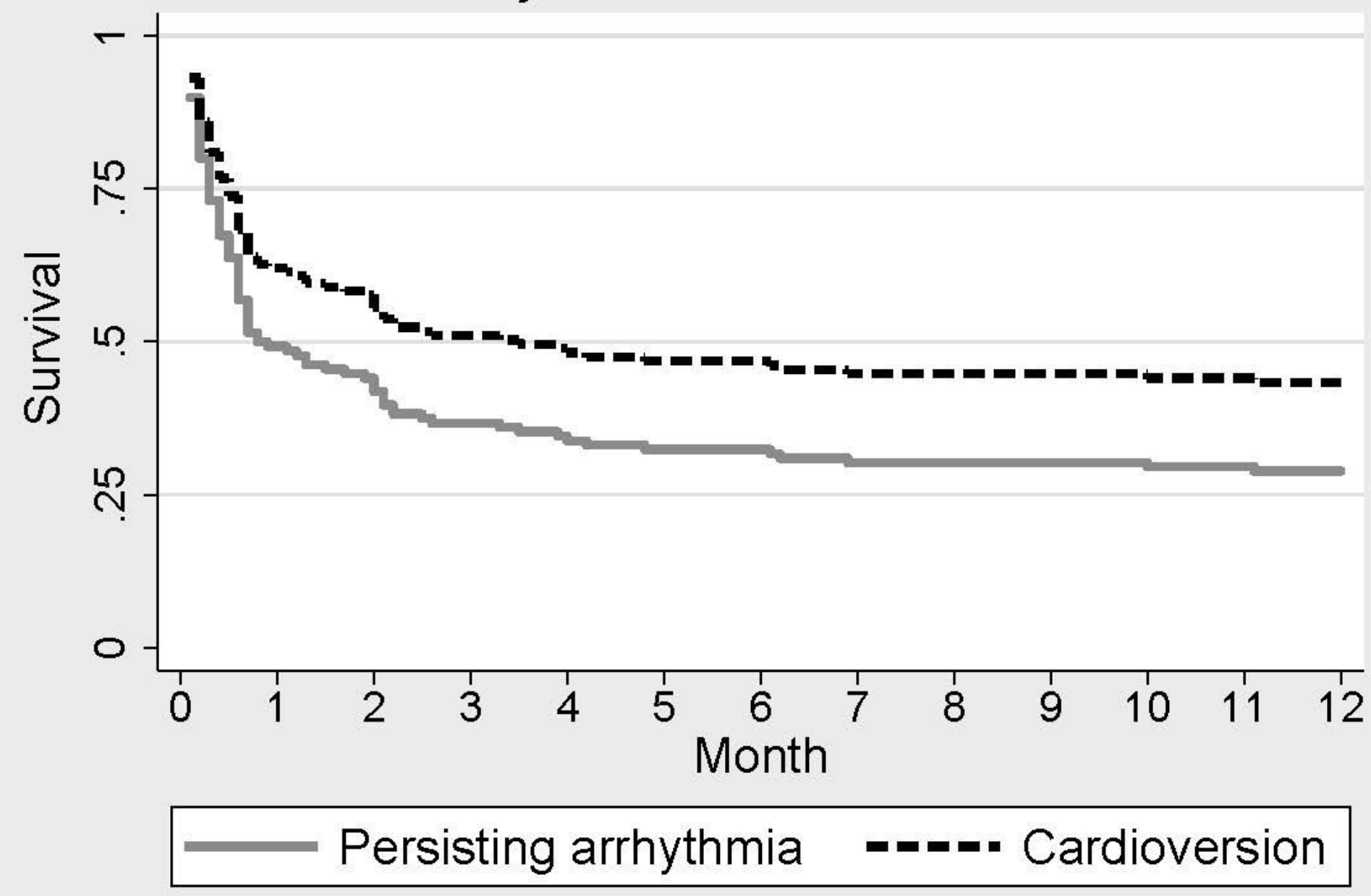

Figure 2

Multivariate analysis showing insignificant 12-month benefit in cardioverting septic shock patients to sinus rhythm (HR0.67,p=0.113). Copied from the author's pilot retrospective study [44]. 
Flowchart of prospective randomized double blinded study of efficacy and safety of $1 \mathrm{c}$ class antiarrhythmic agent (propafenone) in septic shock

Acronym: PRASE - Propafenone versus amiodarone in septic shock

SV arythmia: AF, flutter, SVT...

$\downarrow$

1.) Sepsis and administration of noradrenaline due to hypotension non-responsive to correction of preload

2.) Elevation of arterial lactate $>2.0 \mathrm{mmol} / \mathrm{I}$ during the course of disease, i.e. may be present before or during arrhythmia

3.) Positivity of at least one of the CRP or PCT

4.) Administration of antibiotics for an infectious source

Echocardiography: LV systolic function must be normal or mildly to moderately reduced

\section{Exclusion Criteria:}

- Severe LV systolic dysfunction

- More than 1st degree AV block

- High dose vasopressor therapy with continuous noradrenaline $>1.0 \mathrm{ug} / \mathrm{kg} \cdot \mathrm{min}$

- Known intolerance to amiodarone or propafenone (incl. active thyroid disease other than chronic hormone substitution)

- Absence of septic shock

- Chronic AF - known chronic paroxysmal AF is not an exclusion criterion.

- Dependence on pacemaker

- Status after MAZE procedure

- lodine allergy

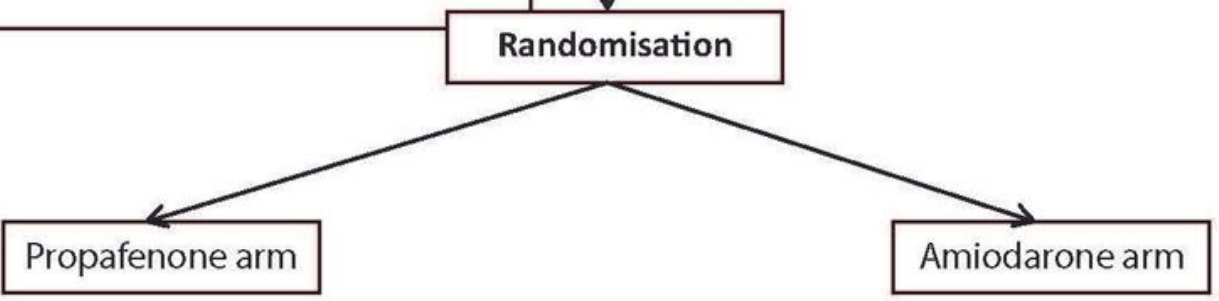

\section{Figure 3}

Flowchart of the study. 


\begin{tabular}{|c|c|c|c|c|c|c|c|c|}
\hline & & & \multicolumn{6}{|c|}{ STUDY PERIOD } \\
\hline & $\begin{array}{l}\text { Screenin } \\
\mathrm{g}\end{array}$ & $\begin{array}{l}\text { Randomi } \\
\text { sation } \\
\text { through } \\
\text { electroni } \\
\text { c CRF }\end{array}$ & \multicolumn{3}{|c|}{ Visits } & $\begin{array}{c}\text { ICU } \\
\text { outcome }\end{array}$ & $\begin{array}{l}\text { 28-days } \\
\text { outcome }\end{array}$ & $\begin{array}{c}12 \mathrm{~m}- \\
\text { outcome }\end{array}$ \\
\hline TIMEPOINT & $-T_{1}$ & 0 & $T+1 h^{*}$ & $T+4 h^{*}$ & $T_{x}^{\text {** }}$ & & & \\
\hline $\begin{array}{r}\text { Septic shock } \\
\text { criteria JAMA } \\
3 / 2016 \\
\end{array}$ & $x$ & & & & & & & \\
\hline Informed consent & $\mathrm{x}$ & & & & & & & \\
\hline Allocation & & $x$ & & & & & & \\
\hline 12-lead ECG & $x$ & & $x$ & $x$ & $x$ & & & \\
\hline $\begin{array}{r}\text { Transthoracic } \\
\text { echocardiography } \\
(\text { TTE) }\end{array}$ & $x$ & & $x$ & $x$ & $x$ & & & \\
\hline $\begin{array}{r}\text { Hemodynamic } \\
\text { assessment }\end{array}$ & $x$ & & $x$ & $x$ & $x$ & $X^{\star \star \star}$ & $X^{\star \star \star}$ & $X^{\star \star \star}$ \\
\hline Laboratory data & $x$ & & & & & & & \\
\hline $\begin{array}{c}\begin{array}{c}\text { Concomitant } \\
\text { medications }\end{array} \\
\end{array}$ & $x$ & & & & & & & \\
\hline INTERVENTIONS: & & & & & & & & \\
\hline Propafenone bolus & & $x$ & & & & & & \\
\hline $\begin{array}{r}\text { Propafenone cont. } \\
\text { infusion }\end{array}$ & & & $x$ & $x$ & $x$ & & & \\
\hline Amiodarone bolus & & $x$ & & & & & & \\
\hline $\begin{array}{r}\text { Amiodarone cont. } \\
\text { infusion }\end{array}$ & & & $x$ & $x$ & $x$ & & & \\
\hline
\end{tabular}

*Visits: 12-lead ECG every $12 \mathrm{~h}$ on infusion, TTE per $24 \mathrm{~h}$ of arrhythmia and $+1 \mathrm{~h}$ after cardioversion, $+4 \mathrm{~h}$ after cardioversion, TTE in any instability

${ }^{\star *} T x$ - day on antiarrhythmic infusion

**AAlive/dead, sinus/persistent arrhythmia

\section{Figure 4}

SPIRIT table for the schedule of enrolment, interventions, and assessments.

\section{Supplementary Files}

This is a list of supplementary files associated with this preprint. Click to download. 
- supplement1.doc

Page 22/22 